\title{
Reduction of Mortality Rate Due to AIDS When Treatment Is Considered
}

\author{
Udoy S. Basak \\ Department of Mathematics, Pabna University of Science \& Technology, Pabna, Bangladesh
}

\section{Email address:}

udoy079@gmail.com

\section{To cite this article:}

Udoy S. Basak. Reduction of Mortality Rate Due to AIDS When Treatment Is Considered. Pure and Applied Mathematics Journal. Vol. 5, No. 4, 2016, pp. 97-102. doi: 10.11648/j.pamj.20160504.12

Received: May 16, 2016; Accepted: May 28, 2016; Published: June 18, 2016

\begin{abstract}
AIDS is one of the most threatening diseases for human being that is caused by a virus named HIV. Here the reduction of the death rate after infected by AIDS has been discussed. A mathematical model of HIV has been formulated. Then its positivity and boundedness has been investigated. It has been shown that it is possible to minimize the mortality rate by providing the treatment to the HIV infected people. Moreover, the control of the transfer rate from the infected class to the AIDS class reduces the disease rate. The increasing of the transfer rate from the infected class to the treated class also reduces the mortality rate.
\end{abstract}

Keywords: HIV, AIDS, Reproduction Number, Endemic Equilibrium Point

\section{Introduction}

In the last decades or so mathematical models in epidemiology are playing a very important role in analyzing the spread and control of the infectious disease that are causing a lot of human death every year. In the past, human being have been threatened by many infectious disease, most of them are caused by viruses [1]. Among them HIV is responsible for a lot of human death. Till now people are dying by this virus.

Human Immunodeficiency Virus (HIV) is a member of Lent Virus sub member family of retrovirus that produces chronic infections in the host and gradually damages the host's immune system [2]. There are two types of HIV viruses: HIV-1 and HIV-2. Among them, HIV-1 is more dangerous. It easily transmits among the people and causes a lot of HIV infections globally every year [3]. Immediately after HIV infection, people cannot determine his/her infection. In this time, HIV infection may not show up on an HIV test, but people who have it are highly infectious and can spread the infection to others. People with chronic HIV infection may not have any HIV-related symptoms or only mild ones [4].

After being infected, people who aren't taking medicine to treat HIV, this period can last a decade or longer, but some may progress through this phase faster. People who are taking medicine to treat HIV the right way, every day may be in this stage for several decades because treatment helps keep the virus in check.

HIV is treated using a combination of medicines to fight HIV infection. This is called antiretroviral therapy (ART). ART isn't a cure, but it can control the virus so that one can live a longer, healthier life and reduce the risk of transmitting HIV to others. ART is recommended for all people with HIV, regardless of how long they've had the virus or how healthy they are. If left untreated, HIV will attack the immune system and eventually progress to AIDS.

\section{Formulation of the Model}

The total sexually-active population at time $t$, denoted by $N(t)$, is subdivided into mutually-exclusive compartments, namely susceptible $(S(t))$, exposed to HIV but show no clinical symptoms of the disease $(E(t))$, HIV infected individuals with clinical symptoms of $\operatorname{HIV}(I(t))$, infected individuals who are taking treatment $(T(t))$, individuals having $\operatorname{AIDS}(A(t))$, so that

$$
N(t)=S(t)+E(t)+I(t)+T(t)+A(t)
$$

Let us suppose that the susceptible population is increasing 
at a constant rate. Let the rate be $\Pi$.

Susceptible individuals acquire HIV infection, following effective contact with people infected with HIV only (i.e. those in the $\mathrm{E}, \mathrm{I}$ and $\mathrm{T}$ classes) at a rate $\lambda$, where

$$
\lambda=\frac{\beta\left(E+\theta_{1} I+\theta_{2} T+\theta_{3} A\right)}{N} \text { [Force of infection for HIV.] }
$$

Here $\beta$ is the transmission rate for $\mathrm{HIV}$ and $\theta_{3}>\theta_{1}>$ $\theta_{2}>1$ are known as the modification parameter. These modification parameters indicate that the infected individuals are more infectious than the treated class. Among these class, AIDS class is assumed to spread more infection than other two classes. In the HIV exposed class $(E(t))$, individuals entering into this class from susceptible class $(S(t))$ at a rate $\lambda$. In the HIV exposed class $(E(t))$ individuals progress to infected class $(I(t))$ at a rate $\omega$. Individual also progresses to the treatment class $(T(t))$ at a rate $\tau_{1}$. And Individual also progresses to the AIDS class $(A(t))$ at a rate $\delta_{1}$.

In the treatment class, individuals progress to AIDS class $(A(t))$ at a rate $\tau_{3}$. Also individuals progress to the AIDS and HIV treatment class $(T(t))$ at a rate $\tau_{4}$. In this class, let $(\mu+d)$ denotes the death rate due to the disease and $\mu$ be the natural mortality rate.

Combining all the aforementioned assumption and definitions, the model becomes:

$$
\begin{aligned}
& \frac{d S}{d t}=\pi-\lambda S-\mu S \\
& \frac{d E}{d t}=\lambda S-\omega E-\mu E \\
& \frac{d I}{d t}=\omega E-\tau_{1} I-\delta_{1} I-\mu I \\
& \frac{d T}{d t}=\tau_{1} I+\tau_{4} A-\tau_{3} T-\mu T \\
& \frac{d A}{d t}=\delta_{1} I-\tau_{4} A+\tau_{3} T-(\mu+d) A
\end{aligned}
$$

Where, $\theta_{3}>\theta_{2}>\theta_{1}>1$.

Schematically this can be shown as follows:

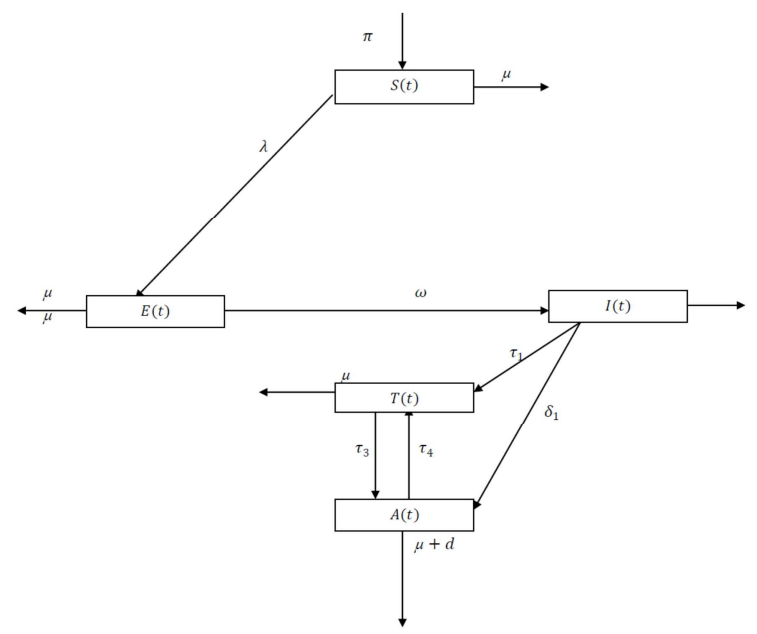

Figure 1. Diagram of the model.

\section{Analysis of the Model}

\subsection{Boundedness Region}

Here we have used some parameters (explained in the table 2) to described the model (1). These variables are nonnegative. Also the state variables (described in table 1) are non-negative for all time $t>0$.

The total population is denoted by $N(t)$. Total population can be written as:

$$
N(t)=S(t)+E(t)+I(t)+T(t)+A(t) .
$$

The equation written above, is changing at a rate

$$
\frac{d N}{d t}=\frac{d S}{d t}+\frac{d E}{d t}+\frac{d I}{d t}+\frac{d T}{d t}+\frac{d A}{d t}=\pi-\mu N-d A
$$

In absence of disease, i.e. for $E=I=T=A=0$, we have

$$
\frac{d N}{d t} \leq \pi-\mu N
$$

By the separation of variables we can write

$$
\frac{d N}{\pi-\mu N} \leq d t
$$

After integration we have

$$
\pi-\mu N \geq C e^{-\mu t}
$$

Here $C$ is the integrating constant which is to be determined. Let at $t=0, N=N_{0}$. From the Eq. (4) we have

$$
C=\pi-\mu N_{0}
$$

Putting the value of $C$ in Eq. (4) we have

$$
\pi-\mu N \geq\left(\pi-\mu N_{0}\right) e^{-\mu t}
$$

$$
\text { As } t \rightarrow \infty, 0 \leq N(t) \leq \frac{\pi}{\mu}
$$

Therefore, the region $\Psi=\left\{(S, E, I, T, A) \in \mathfrak{R}_{+}^{5}: N \leq \frac{\pi}{\mu}\right\}$ contains the feasible solution of the model (1).

\subsection{Positivity of Solution}

Now we need to check the positivity of the solution of the model (1). Let the initial data be $\{S(0), E(0), I(0), T(0), A(0) \in$ $\Psi\}$. Now we have to show that the solution set $\{S(t), E(t), I(t), T(t), A(t)\}$ of the system (1) is positive.

Consider the $1^{\text {st }}$ equation of the model (1)

$$
\frac{d S}{d t}=\pi-\lambda S-\mu S>-(\lambda+\mu) S \Rightarrow \frac{d S}{d t}>-(\lambda+\mu) S
$$

After integration we can write

$S(t)>S(0) e^{\int-(\lambda+\mu) d t}>0$ provided $(\lambda+\mu)<\infty$.

Similarly from the $2^{\text {nd }}, 3^{\text {rd }}, 4^{\text {th }}$ and $5^{\text {th }}$ equations respectively we have

$E(t)>E(0) e^{\int-(\omega+\mu) d t}>0$ provided $(\omega+\mu)<\infty$.

$I(t)>I(0) e^{\int-\left(\tau_{1}+\delta_{1}+\mu\right) d t}>0$ provided $\left(\tau_{1}+\delta_{1}+\mu\right)<\infty$.

$T(t)>T(0) e^{\int-\left(\tau_{3}+\mu\right) d t}>0$ provided $\left(\tau_{3}+\mu\right)<\infty$.

$A(t)>A(0) e^{\int-\left(\tau_{4}+\mu+d\right) d t}>0$ provided $\left(\tau_{4}+\mu+d\right)<\infty$. 
Thus the solution set $\{S(t), E(t), I(t), T(t), A(t)\}$ is positive for $t>0$.

\section{Disease-Free Equilibrium Point}

Disease free equilibrium point is denoted by $E_{0}$. To obtain the disease-free equilibrium points on $\Psi \cap \mathfrak{R}_{+}^{5}$, we need to put $E=I=T=A=0$ (since in the disease-free equilibrium there is no disease). The positive DFE for this model is $N=\frac{\pi}{\mu}$. So we can conclude that the model has a disease-free equilibrium point and the disease-free equilibrium point of this model is $E_{0}=\left(\frac{\pi}{\mu}, 0,0,0,0\right)$.

\section{Local Stability of the Disease-Free Equilibrium}

The basic reproduction number which is denoted by $R_{0}$ plays a vital role in the stability of the model. The number of new infections generated by an infected individual is known as the basic reproduction number. The associated nonnegative matrix $F$ for the new infection terms and the non-singular $M$-matrix, $V$ for the remaining transfer terms are given respectively by

$$
F=\left(\begin{array}{cccc}
\beta & \beta \theta_{1} & \beta \theta_{2} & \beta \theta_{3} \\
0 & 0 & 0 & 0 \\
0 & 0 & 0 & 0 \\
0 & 0 & 0 & 0
\end{array}\right)
$$

And $V$ has the following expression

$$
V=\left(\begin{array}{cccc}
\omega+\mu & 0 & 0 & 0 \\
0 & -\omega+\tau_{1}+\delta_{1}+\mu & 0 & 0 \\
0 & -\tau_{1} & \mu+\tau_{3} & -\tau_{4} \\
0 & \delta_{1} & -\tau_{3} & \tau_{4}+(\mu+d)
\end{array}\right)
$$

The basic reproduction number $R_{0}$ is the spectral radius of the matrix $F V^{-1}$. The eigenvalues of the matrix $F V^{-1}$ are

$$
\begin{aligned}
R_{0}=\left[\beta \left(\theta_{1} \omega \tau_{4} \mu\right.\right. & +\theta_{1} \omega \mu \tau_{3}+\theta_{1} \omega d \mu+\theta_{1} \omega d \tau_{3}+\theta_{2} \omega \tau_{1} \mu+\theta_{2} \omega \tau_{1} d+\theta_{2} \omega \tau_{4} \delta_{1}+\theta_{2} \omega \tau_{4} \tau_{1}+\theta_{3} \omega \delta_{1} \mu+\theta_{3} \omega \delta_{1} \tau_{3}+\theta_{3} \omega \tau_{3} \tau_{1} \\
& +\tau_{1} \tau_{4} \mu+\tau_{1} \mu \tau_{3}+\tau_{1} d \mu+\tau_{1} d \tau_{3}+\tau_{4} \delta_{1} \mu+\tau_{1} \mu+\delta_{1} \mu^{2}+\tau_{4} \mu^{2}+\mu^{2} \tau_{3}+d \mu^{2}+\delta_{1} \mu \tau_{3}+\delta_{1} d \mu+\delta_{1} d \tau_{3}+\mu d \tau_{3} \\
& \left.+\theta_{1} \omega \mu^{2}+\mu^{3}\right) /\left(\tau_{1} \omega \tau_{4} \mu+\tau_{1} \omega \mu \tau_{3}+\tau_{1} \omega d \mu+\delta_{1} \omega \mu^{2}+\omega \mu^{3}+\tau_{1} \mu^{3}+\delta_{1} \mu^{3}+\mu^{3} \tau^{4}+\mu^{3} \tau_{3}+\mu^{3} d+\tau_{1} \omega \mu^{2}\right. \\
& +\omega \mu^{2} \tau^{4}+\omega \mu^{2} \tau_{3}+\omega \mu^{2} d+\tau_{1} \mu^{2} \tau_{4}+\tau_{1} \mu^{2} \tau_{3}+\tau_{1} \mu^{2} d+\delta_{1} \mu^{2} \tau_{4}+\delta_{1} \mu^{2} \tau_{3}+\delta_{1} \mu^{2} d+\mu^{2} d \tau_{3}+\mu^{4}+\tau_{1} \omega d \tau_{3} \\
& \left.\left.+\delta_{1} \omega \tau_{4} \mu+\delta_{1} \omega \mu \tau_{3}+\delta_{1} \omega d \mu+\delta_{1} \omega d \tau_{3}+\omega \mu d \tau_{3}+\tau_{1} \mu d \tau_{3}+\delta_{1} \mu d \tau_{3}\right)\right]
\end{aligned}
$$

Lemma [5]. Local stability of the disease-free equilibrium depends on the basic reproduction number. If the basic reproduction number is bigger than one, then disease-free equilibrium point locally asymptotically unstable and if the basic reproduction number is less than one, then it is locally asymptotically unstable.

\section{Global Stability of the Disease-Free Equilibrium}

Here we have used a technique established by CastilloChavez [6] to investigate the global stability of the diseasefree equilibrium point. We rewrite the model as:

$$
\begin{aligned}
& \frac{d X}{d t}=H(X, Z) \\
& \frac{d Z}{d t}=G(X, Z), G(X, 0)=0
\end{aligned}
$$

Where $X=S$ and $Z=(E, I, T, A$, ). Here the components of $Z \in \mathbb{R}_{+}^{4}$ denotes the infected population. The disease free equilibrium is now denoted as:

$$
E_{0}=\left(X^{*}, 0\right), X^{*}=\frac{\pi}{\mu}
$$

The condition must be met to guarantee a local asymptotic stability:

$$
\frac{d X}{d t}=H(X, 0)
$$

Here, $X^{*}$ is globally asymptotically stable (GAS).

$G(X, Z)=P Z-G^{*}(X, Z), G^{*}(X, Z), \geq$ of or $(X, Z) \in \Omega$

Where $P=D_{Z} G\left(X^{*}, 0\right)$, is an $M$-matrix (the off-diagonal elements of $P$ are non-negative) and $\Omega$ is the region where the model makes biological sense. If the system satisfies the conditions of then the theorem below holds:

Theorem [5]. The fixed point $E_{0}=\left(X^{*}, 0\right)$ is a globally asymptotically stable equilibrium of the system (6) provided that $R_{0}<1$ and the assumptions in (4) are satisfied.

Proof. Form the model system (1) and (6), we have

$$
\begin{gathered}
H(X, 0)=(\pi-\mu S) \\
G(X, Z)=P Z-G^{*}(X, Z)
\end{gathered}
$$

Where

$$
G^{*}(X, Z)=\left(\begin{array}{c}
\beta\left[E+\theta_{1} I+\theta_{2} T+\theta_{3} A\right]\left[1-\frac{S}{N}\right] \\
0 \\
0 \\
0
\end{array}\right)=\left(\begin{array}{c}
G_{1}^{*}(X, Z) \\
G_{2}^{*}(X, Z) \\
G_{3}^{*}(X, Z) \\
G_{4}^{*}(X, Z)
\end{array}\right)
$$

Here $G_{1}^{*}>0 \&\left(G_{2}^{*}, G_{3}^{*}, G_{4}^{*}\right)=0$

So the conditions are fulfilled. So $E^{*}$ is globally asymptotically stable when $R_{0}<1$.

\section{Endemic Equilibrium of the Model}

We have used a well-known technique known as the Central Manifold Theorem [6] to check the stability of the 
endemic equilibrium point of the model. To apply this theorem we make the following change of variables.

Let

$$
\begin{gathered}
\mathrm{S}=\mathrm{x}_{1}, \mathrm{E}=\mathrm{x}_{2}, \mathrm{I}=\mathrm{x}_{3}, \mathrm{~T}=\mathrm{x}_{4}, \mathrm{~A}=\mathrm{x}_{5} \\
N=x_{1}+x_{2}+x_{3}+x_{4}+x_{5}
\end{gathered}
$$

The model (1) can be rewritten in the form:

$$
\frac{d S}{d t}=f(x)
$$

Where $X=\left(x_{1}, x_{2}, x_{3}, x_{4}, x_{5}\right)$, and $F=\left(f_{1}, f_{2}, f_{3}, f_{4}, f_{5},\right)$ as

$$
\begin{aligned}
& f_{1}=\pi-\lambda x_{1}-\mu x_{1} \\
& f_{2}=\lambda x_{1}-\omega x_{2}-\mu x_{2} \\
& f_{3}=\omega x_{2}-\tau_{1} x_{3}-\delta_{1} x_{3}-\mu_{3} \\
& f_{4}=\tau_{1} x_{3}+\tau_{4} x_{5}-\tau_{3} x_{4}-\mu x_{4} \\
& f_{5}=\delta_{1} x_{3}-\tau_{4} x_{5}+\tau_{3} x_{4}-(\mu+d) x_{5}
\end{aligned}
$$

Where $\lambda=\frac{\beta\left(x_{2}+\theta_{1} x_{3}+\theta_{2} x_{4}+\theta_{3} x_{5}\right)}{x_{1}+x_{2}+x_{3}+x_{4}+x_{5}}$

The Jacobean of the system (8) is

$$
J=\left[\begin{array}{ccccc}
-\mu & -\beta & -\beta \theta_{1} & -\beta \theta_{2} & -\beta \theta_{3} \\
0 & -\beta-\mu-\omega & \beta \theta_{1} & \beta \theta_{2} & \beta \theta_{3} \\
0 & \omega & -\tau_{1}-\delta_{1}-\mu & 0 & 0 \\
0 & 0 & \tau_{1} & -\tau_{3}-\mu & \tau_{4} \\
0 & 0 & \delta_{1} & \tau_{3} & -\tau_{4}-\mu-d
\end{array}\right]
$$

To analyze the dynamics of (8), we compute the eigenvalues of the Jacobian of (8) at the disease free equilibrium (DFE). It can be shown that this Jacobian has a right eigenvector given by:

$$
V=\left(V_{1}, V_{2}, V_{3}, V_{4}, V_{5}\right)^{T}
$$

Where

$$
\begin{aligned}
& V_{1}=-\frac{\beta\left(V_{2}+\theta_{1} V_{3}+\theta_{2} V_{4}+\theta_{3} V_{5}\right)}{\mu} \\
& V_{2}=\frac{\left(\tau_{1}+\delta_{1}+\mu\right) V_{3}}{\omega} \\
& V_{3}=V_{3} \\
& V_{4}=\frac{\tau_{1} V_{3}+\tau_{4} V_{5}}{\tau_{3}+\mu} \\
& V_{5}=\frac{\delta_{1} V_{3}+\tau_{3} V_{4}}{\tau_{4}+\mu+d}
\end{aligned}
$$

And the left eigenvectors are given by

$$
\begin{aligned}
& W=\left(w_{1}, w_{2}, w_{3}, w_{4}, w_{5}\right)^{\mathrm{T}} \\
& W_{1}=0 \\
& W_{2}=\frac{\omega W_{3}}{\mu+\omega-\beta}
\end{aligned}
$$

$$
\begin{aligned}
& W_{3}=W_{3} \\
& W_{4}=\frac{\tau_{3} W_{5}(\beta-\omega-\mu)-\beta \omega \theta_{2} W_{3}}{\left(\tau_{3}+\mu\right)(\beta-\omega-\mu)} \\
& W_{5}=\frac{\beta \theta_{3} W_{2}+\tau_{4} W_{4}}{\tau_{4}+\mu+d}
\end{aligned}
$$

It can be shown after some manipulations involving the evaluation of the associated non-vanishing partial derivatives of $f$ that

$$
\begin{aligned}
& \qquad \begin{aligned}
S^{*}=\frac{2 \beta}{N}\left(W_{2}\right. & \left.+W_{3}+W_{4}+W_{5}\right)\left(W_{2}+W_{4} \theta_{2}+W_{5} \theta_{3}\right. \\
& \left.+W_{3} \theta_{1}\right)\left(V_{1}-V_{2}\right)
\end{aligned} \\
& \text { And } r^{*}=-\left(W_{2}+W_{4} \theta_{2}+W_{5} \theta_{3}+W_{3} \theta_{1}\right)\left(V_{1}-V_{2}\right)
\end{aligned}
$$

Since $v_{1}=-\frac{\beta\left(v_{2}+\theta_{1} v_{3}+\theta_{2} v_{4}+\theta_{3} v_{5}\right)}{\mu}$ which is less than zero and $v_{2}=\frac{\left(\tau_{1}+\delta_{1}+\mu\right) v_{3}}{\omega}$ which is positive.

So $v_{1}-v_{2}<0$.

Thus we have

$$
\begin{gathered}
S^{*}=\frac{2 \beta}{N}\left(w_{2}+w_{3}+w_{4}+w_{5}\right)\left(w_{2}+w \theta_{2}+w_{5} \theta_{3}\right. \\
\left.+w_{3} \theta_{1}\right)\left(v_{1}-v_{2}\right)<0
\end{gathered}
$$

Again $w_{1}, w_{2}, w_{3}, w_{4}, w_{5}$ are all positive and all the parameters are positive. So

$$
r^{*}=-\left(w_{2}+w_{4} \theta_{2}+w_{5} \theta_{3}+w_{3} \theta_{1}\right)\left(v_{1}-v_{2}\right)>0
$$

Thus we have established the following theorem:

Theorem. Thus we have the following results: the endemic equilibrium point is locally stable when the basic reproduction number is bigger than one and unstable when basic reproduction number is less than one.

\section{Numerical Simulations and Discussions}

The description of the variable is shown in the Table 1 . In the model we have used some parameters. The values of the parameters that are used in the model are shown in the table 2.

Table 1. Description of variables of the model.

\begin{tabular}{ll}
\hline Variables & Descriptions \\
\hline$S(t)$ & Susceptible class \\
$E(t)$ & Individuals Exposed to AIDS but show no clinical symptoms. \\
$I(t)$ & Individuals infected with AIDS not yet displaying symptoms. \\
$T(t)$ & HIV infected individuals are in Treatment Class \\
$A(t)$ & HIV infected individuals and displaying symptoms of AIDS. \\
\hline
\end{tabular}

Table 2. The values of the parameters of the model.

\begin{tabular}{lll}
\hline Variables & Description & Values \\
\hline$\Pi$ & Recruitment rate of humans & $0.00384[11]$ \\
$\beta$ & Modification Parameter & 0.0082 \\
\hline
\end{tabular}




\begin{tabular}{lll}
\hline Variables & Description & Values \\
\hline$\theta_{1}$ & Modification Parameter & 1.2 \\
$\theta_{2}$ & Modification Parameter & 1.4 \\
$\theta_{3}$ & Modification Parameter & 1.5 \\
$\delta_{1}$ & Transfer rate from $I(t)$ to $A(t)$ & $0.025[6]$ \\
$\omega$ & Transfer rate from $E(t)$ to $I(t)$ & $0.03[8]$ \\
$\tau_{1}$ & Transfer rate from $I(t)$ to $T(t)$ & $0.04[4]$ \\
$\tau_{3}$ & Transfer rate from $T(t)$ to $A(t)$ & $0.025[4]$ \\
$\tau_{4}$ & Transfer rate from $A(t)$ to $T(t)$ & $0.02[5]$ \\
$\mu$ & Natural Death & $0.0003[11]$ \\
$d$ & Death rate after having AIDS & $0.003[11]$ \\
\hline
\end{tabular}

Figure 2 shows that the total infected population is increasing when the basic reproduction number $R_{0}>1$ and figure 3 shows that the infected population is decreasing when $R_{0}<1$. Here the value of the basic reproduction number $R_{0}$ depends on the transfer rate $\tau_{1}$ from the infected class $I(t)$ to treated class $T(t)$. The values of the reproduction number $R_{0}$ decreases with the transfer rate $\tau_{1}$. Thus we can minimize the mortality rate by increasing the number of the treated population. Figure 4 shows that, the prevalence is decreasing when the basic reproduction number $R_{0}<1$ and figure 5 shows that it is increasing when $R_{0}>1$.

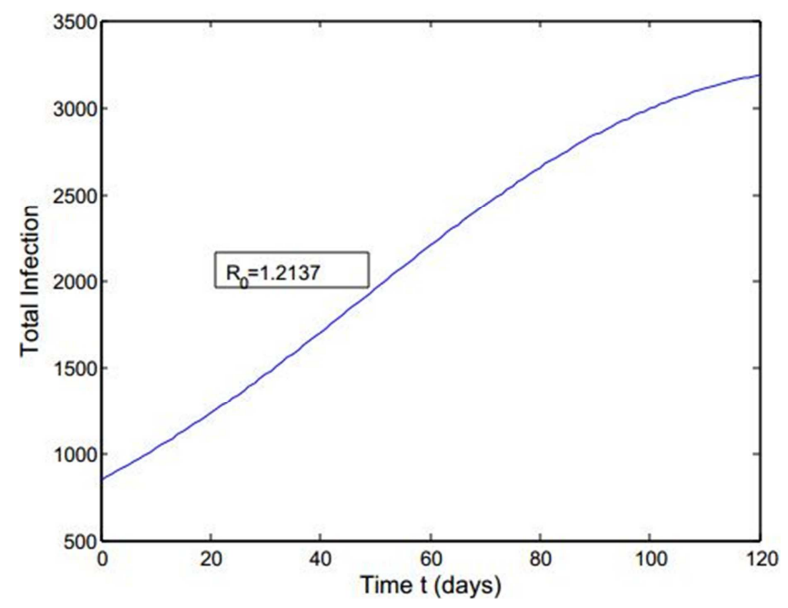

Figure 2. Total infection when $R_{0}>1$.

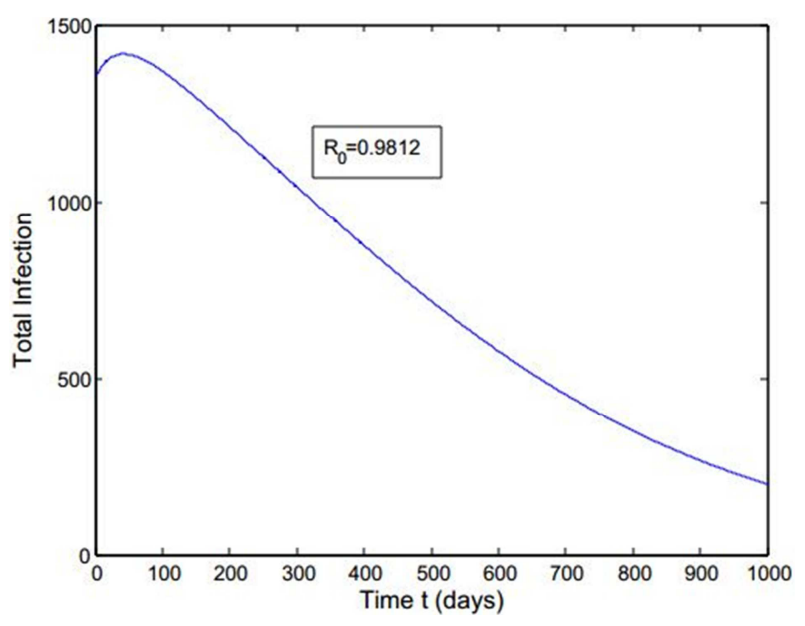

Figure 3. Total Infection when $R_{0}<1$.

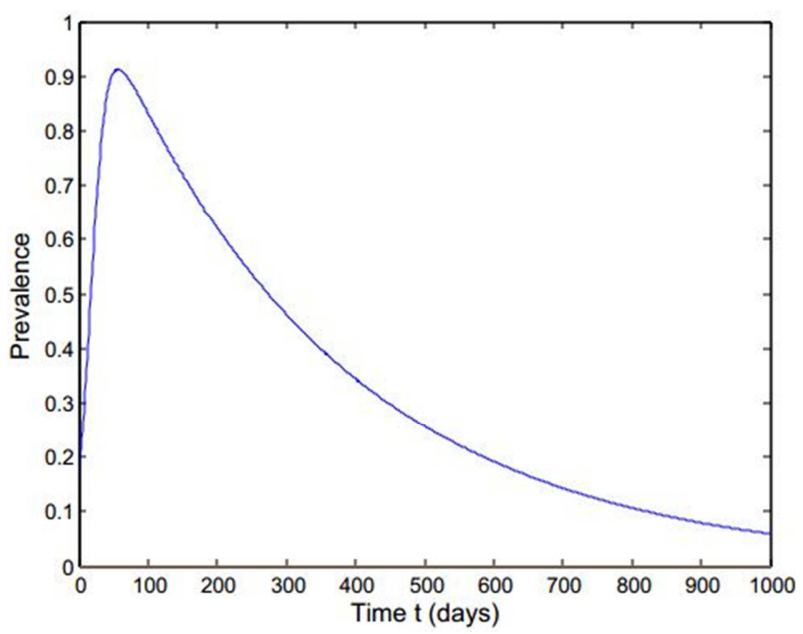

Figure 4. Prevalence when $R_{0}<1$.

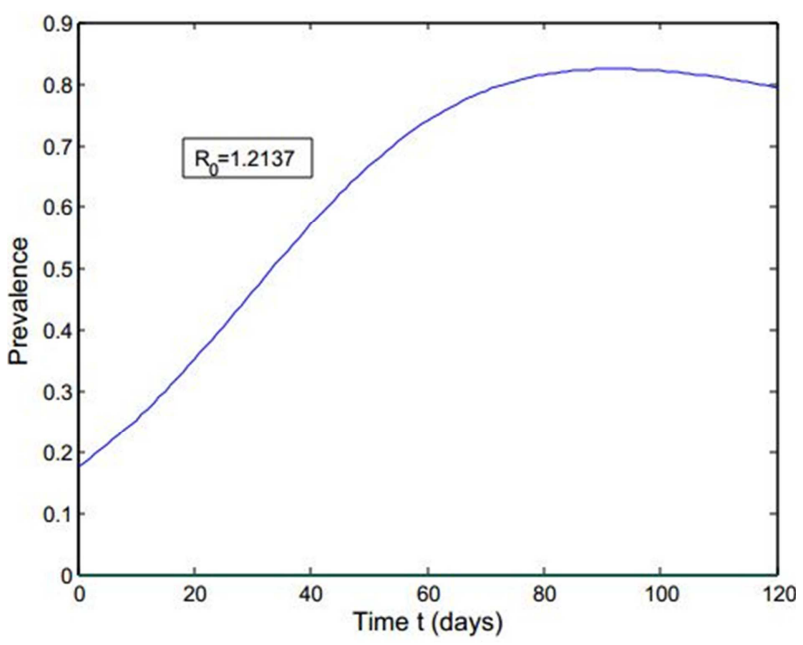

Figure 5. Prevalence when $R_{0}>1$.

\section{Conclusions}

AIDS is one of the devastating diseases for human being. Mathematical models are used to estimate the dynamics of the diseases. Here we have formulated a model describing the HIV with treatment. The findings of the study are listed below:

- We can minimize the mortality rate by providing the treatment to the HIV infected people.

- The basic reproduction number highly depends on $\delta_{1}$, which is the transfer rate from $I(t)$ to $A(t)$ class. Thus the reduction in the transfer rate from the infected rate $I(t)$ to the AIDS class $A(t)$ will reduce the mortality rate.

- The increasing of the transfer rate $\tau_{1}$ from the infected class $I(t)$ to the treated class $T(t)$ also reduces the disease burden.

\section{References}

[1] F. Brauer and C. Castillo-Chavez, Mathematical Models in Population Biology and Epidemiology, Springer-Verlag. New York, 2001. 
[2] Hu et al (1996), "The emerging genetic diversity of HIV, the importance of global surveillance for diagnostic research and prevention”. JAMA, 275 (3), 210-216.

[3] Reeves JD, Doms RW (2002). "Human immunodeficiency virus type 2". The Journal of general virology 83 (Pt 6): 125365. doi:10.1099/vir.0.18253-0 (inactive 2015-01-13). PMID 12029140 .

[4] Santiago et al, "Simian Immunodeficiency Virus Infection in Free-Ranging Sooty Mangabeys (Cercocebus atys atys) from the Tai Forest, Cote d'Ivoire: Implications for the Origin of Epidemic Human Immunodeficiency Virus Type 2", Journal of Virology 79 (19): 12515-27.

[5] Chavez et al (2004), "Dynamical model of tuberculosis and their applications". Math. Bioscience., 1: 361-404.

[6] Lawi et al (2011), "Mathematical model for malaria and meningitis co-infection among children". Applied Mathematics Sciences, Vol. 5, 2011, no. 47, 2337-2359.
[7] Weiss RA (May 1993). "How does HIV cause AIDS?" Science 260(5112): 1273-1279.

[8] Douek DC, Roederer M, Koup RA (2009). Emerging Concepts in the Immunopathogenesis of AIDS." Annu. Rev. Med. 60: 471-84.

[9] Reeves JD, Doms RW (2002). Human immunodeficiency virus type 2." The Journal of general virology 83 (Pt 6): 1253-65. doi:10.1099/vir.0.18253-0 (inactive 2015-01-13). PMID 12029140 .

[10] Garg H, Mohl J, Joshi A (Nov 9, 2012). HIV-1 induced bystander apoptosis." Viruses 4 (11): 3020-43. doi: 10.3390/v4113020. PMC 3509682. PMID 23202514.

[11] Udoy S. Basak et al. "Mathematical Analysis of an HIV/AIDS Epidemic Model". American Journal of Mathematics and Statistics 2015, 5(5): 253-258 DOI: 10.5923/j.ajms.20150505.05. 